\title{
Natural Regression of Frailty Among Community-Dwelling Older Adults: A Systematic Review and Meta-Analysis
}

\author{
Richard Ofori-Asenso, MSc, ${ }^{1}$ Ken Lee Chin, PhD, ${ }^{1,2}$ Mohsen Mazidi, PhD, ${ }^{3,4}$ \\ Ella Zomer, PhD, ${ }^{1}$ Jenni llomaki, PhD, ${ }^{1,5}$ Zanfina Ademi, PhD, ${ }^{1}$ J Simon Bell, PhD, ${ }^{1,5,6}$ and \\ Danny Liew, PhD ${ }^{1, *}$
}

'Department of Epidemiology and Preventive Medicine, Monash University, Melbourne, Victoria, Australia. ${ }^{2}$ Department of Medical Education, Melbourne Medical School, The University of Melbourne, Victoria, Australia. ${ }^{3}$ Key State Laboratory of Molecular Developmental Biology, Institute of Genetics and Developmental Biology, Chinese Academy of Sciences, Chaoyang, China. ${ }^{4}$ Institute of Genetics and Developmental Biology, International College, University of Chinese Academy of Science (IC-UCAS), Chaoyang, China. ${ }^{5}$ Centre for Medicine Use and Safety, Faculty of Pharmacy and Pharmaceutical Sciences, Monash University, Melbourne, Victoria, Australia. ${ }^{6}$ Centre for Research Excellence in Frailty and Healthy Ageing, University of Adelaide, South Australia, Australia.

\begin{abstract}
*Address Correspondence to: Danny Liew, PhD, Department of Epidemiology and Preventive Medicine, Monash University, 553 St Kilda Road, VIC 3004, Melbourne, Victoria, Australia. E-mail: danny.liew@monash.edu
\end{abstract}

Received: January 29, 2019; Editorial Decision Date: April 11, 2019

Decision Editor: Patricia C Heyn, PhD

\begin{abstract}
Background and Objectives: Frailty is a dynamic process with potential transitions over time. However, there is limited understanding of the patterns of frailty improvement. We conducted a systematic review and meta-analysis to estimate the natural rate of frailty regression among community-dwelling older adults aged at least 60 years.

Research Design and Methods: Systematic searches for studies reporting frailty improvement were performed in 5 databases (Medline, Embase, CINAHL plus, Web of Science, and PsycINFO) from inception until January 2019.

Results: Twenty-five studies from 26 countries were included. Among a baseline population of more than 50,000 individuals, the pooled prevalence of pre-frailty and frailty was 50.5\% (95\% confidence interval [CI] $47.8-53.3)$ and $12.8 \%(95 \%$ CI 9.1-17.0), respectively. During a median follow-up of 3.0 (range 1-10.0) years, $23.3 \%$ of surviving pre-frail individuals regressed to a robust state and $35.2 \%$ of surviving frail individuals reversed to a pre-frail or robust state. The pooled remission rates among people with pre-frailty and frailty were 80.4 (95\% CI 61.7-104.6) and 135.3 (95\% CI 98.1-186.5) per 1,000 person-years, respectively. Frailty and pre-frailty improvement rates varied by sex, diagnostic criteria, study region, and follow-up duration. The remission rates were significantly reduced when accounting for progressions to death. The heterogeneity of included studies was high which reflected considerable differences in methodological approach.

Discussion and Implications: Although frailty is highly prevalent in older people, natural remission is possible and common. Improved understanding of the factors that confer increased likelihood of frailty regression may support the design of interventions to reduce the burden of frailty.
\end{abstract}

Keywords: Pre-frailty, Physical phenotype, Deficit accumulation, Health status

Frailty, a complex phenomenon distinct from disability and comorbidity, which results from a decline in reserve capacity, affecting multiple physiological systems, is a major issue among older adults (Clegg, Young, Iliffe, Rikkert, \& Rockwood, 2013; Xue, 2011). At present, there are no universally accepted criteria nor clinical definitions for frailty 
(Dent, Kowal, \& Hoogendijk, 2016). Nonetheless, two main theoretical concepts underpin the assessment of frailty: phenotype of frailty and accumulation of deficits. The frailty phenotype as described by Fried and colleagues, is based on five-predefined physical components: weight loss, exhaustion, weakness, slowness, and low physical activity, with frailty diagnosed as the presence of three or more of these components (Fried et al., 2001). Rockwood and colleagues also conceptualized frailty as an accumulation of deficits (symptoms, signs, functional impairment, and laboratory abnormalities), and demonstrated that more deficits confer greater risk (Rockwood et al., 1999). Both the Fried criteria and the accumulation of deficits approach have been extensively validated and are widely used (Cesari, Gambassi, van Kan, \& Vellas, 2014; Hubbard, O’Mahony, \& Woodhouse, 2009). The frailty phenotype is commonly used because it allows for better clinical operationalization in a variety of health care practice settings, whereas the deficit accumulation approach is also used because it has a strong relationship with mortality and allows for different combinations of deficits that all predict mortality equivalently well (Dent et al., 2016; Malmstrom, Miller, \& Morley, 2014).

Frailty is usually considered as a three-staged process: robust, pre-frail, and frail (Xue, 2011). In the case of the Fried five-phenotype criteria, individuals are characterized as robust, pre-frail, and frail if $0,1-2$, and at least 3 of the criteria are met, respectively (Fried et al., 2001). For the accumulation of deficits approach, variable cut-offs have been used to define frailty in the literature (Aguayo et al., 2017). A systematic review by Collard and colleagues, reported a prevalence of $9.9 \%$ (95\% confidence interval [CI $9.6-10.2)$ and $13.6 \%$ (95\% CI 13.2-14.0) for frailty in community-dwelling adults aged 65 years and older in high-income countries based on the Fried's definition and the frailty index, respectively (Collard, Boter, Schoevers, \& Oude Voshaar, 2012). Increasingly, frailty has been associated with adverse health outcomes such as falls (Cheng \& Chang, 2017; Kojima, 2015), delirium (Persico et al., 2018), hospitalization (Chang, Lin, \& Cheng, 2018; Kojima, 2016), institutionalization (Kojima, 2018), incident disability (Kojima, 2017; Vermeiren et al., 2016), and death (Chang \& Lin, 2015; Kojima, Iliffe, \& Walters, 2018), as well as being associated with higher health care costs (Bock et al., 2016). Despite the increased recognition of the importance of early identification and management of frailty (Dent et al., 2017; Turner, Clegg, British Geriatrics, Age, \& Royal College of General, 2014), there is considerable debate about frailty screening particularly related to who should be screened and where and when (Ambagtsheer et al., 2019).

Frailty is considered a dynamic process, with potential fluctuations over time (Lang, Michel, \& Zekry, 2009; Xue, 2011). Nonetheless, the epidemiological data on frailty remain dominated by a focus on its burden and progression with less attention to the patterns of frailty improvement. Recently, there has been increased interest in understanding the various transition patterns among people with frailty (Michel,
Cruz-Jentoft, \& Cederholm, 2015; O’Caoimh et al., 2018). Kojima and colleagues, for example, reported a systematic review and meta-analysis describing the patterns of frailty transitions among adults aged 50 years and over based on data from 16 studies (Kojima, Taniguchi, Iliffe, Jivraj, \& Walters, 2019). However, their analysis focused only on studies using the Frailty phenotype and reported the pooled proportion of people who regressed with no indication of how quickly remission occurs (i.e., remission rate). In addition, there has been limited comparison of the rates of frailty improvement across different regions or country income levels.

Greater insight of the extent of frailty improvement may benefit clinical decision making as well as informing the design and targeting of public health strategies (BandeenRoche \& Espinoza, 2017). Thus, we conducted a systematic review to summarize the available epidemiological data on the rate of frailty improvement among communitydwelling older adults aged 60 years and older.

\section{Methods}

We undertook a systematic review in accordance with the PRISMA (Preferred Reporting Items for Systematic Reviews and Meta-Analyses) guidelines (Moher, Liberati, Tetzlaff, Altman, \& Group, 2009) (Supplementary Table 1 for checklist). The review protocol was registered in PROSPERO (International Prospective Register of Systematic Reviews); CRD42019121303.

\section{Search Strategy and Selection Criteria}

We searched Medline, Embase, CINAHL Plus, Web of Science, and PsycINFO for studies reporting on frailty remission. The searches were first performed on December 10, 2018 and updated on a weekly basis until January 29, 2019 so as to enable the tracking of new publications. The keywords used in the search were "frailty or frailty syndrome or geriatric disorders" AND "elderly or older or senior" AND "transition or trajectory or improvement or remission". No language restrictions were imposed. Supplementary Table 2 presents the search strategies used for the various databases. Reference lists of the relevant articles were also hand searched for additional studies.

Cohort studies were considered potentially eligible if they examined frailty regression (transition from more to less severe frailty states) among community-dwelling older adults aged 60 years and older at baseline in the absence of interventions (i.e., natural regression of frailty). We selected the 60 years cut-off to be consistent with the United Nations' definition of older persons (Department of Economic and Social Affairs Population Division, 2013). Studies must have measured frailty using a validated method, such as the Fried phenotype criteria or the frailty index (Fried et al., 2001; Rockwood et al., 1999).

Studies were excluded if they did not assess or report data on frailty improvement or involved participants selected on the basis of an index disease. Studies involving participants 
across the age spectrum were excluded unless there were data reported specifically for people aged 60 years and older at baseline. Studies involving hospitalized or institutionalized people were excluded. Similarly, studies reporting only changes in mean frailty scores or those using statistical methods such as group-based trajectory modeling were excluded because these studies were designed primarily to identify groups of individuals following approximately the same frailty trajectories. Randomized controlled trials, reviews, conference abstracts, editorials, and commentaries were also excluded.

All potentially eligible studies identified were searched for duplicates using Endnote X7, followed by title, abstract, and full-text assessments. In the case of multiple studies reporting on the same cohort, the study with the more detailed information or sample size was selected. Title and abstract screening were performed independently by two reviewers (R. Ofori-Asenso and K. L. Chin) and the list of studies selected for full text assessment were cross-checked for consistency. All articles selected for full text evaluation were also screened by two reviewers (R. Ofori-Asenso and K. L. Chin) independently. A prespecified procedure involving adjudication by a third reviewer (D. Liew) was in place to address potential disagreements (related to study selection) between the reviewers. However, this mechanism was never used as no disagreements occurred.

\section{Data Extraction}

The data extraction was performed by R. Ofori-Asenso and cross-checked by K. L. Chin. For each included study, the following information was collected: author details, country, study cohort name if any, publication year, sample size, age range at baseline, frailty assessment method, duration of follow-up, and data on frailty improvement. If available, age- and sex-specific remission data were collected. To highlight the burden of frailty and pre-frailty, we also collected data on the baseline prevalence of frailty and pre-frailty where available. Study authors were contacted for additional data where necessary.

\section{Methodological Quality Assessment}

Two reviewers (R. Ofori-Asenso and K. L. Chin) evaluated the methodological quality of studies using the nine items of the Joanna Briggs Institute (JBI)'s critical assessment tool for prevalence and incidence studies (Munn, Moola, Lisy, Riitano, \& Tufanaru, 2015). A study was considered ineligible if it met less than five of the criteria.

\section{Statistical Analysis}

For each study, we extracted or calculated the rate of frailty improvement (transition from severe to less severe state) per 1,000 person-years based on the event rates and the mean duration of follow-up (Jager, Zoccali, Kramar, \& Dekker, 2007; Yousef et al., 2008). Some studies used a $100 \%$ survivor cohort (i.e., excluded people who died) over the duration of the study. As such, for studies that included those who died in the estimation of remission rate, we recalculated the transition rate by restricting the sample to the fully surviving cohort with frailty assessment data at follow-up. This approach was intended to improve comparability across studies so as to minimize the impact of survivorship bias (Delgado-Rodriguez \& Llorca, 2004). To derive 95\% CIs of event rates, the exact methods, based on the Poisson distribution was adopted. If studies reported zero number of remissions a correction of 0.5 was applied to the observed events and person-years to allow for the calculation of CIs (Sutton, Abrams, Jones, Sheldon, \& Song, 2000).

A meta-analysis was performed using the log-transformed transition rates and corresponding 95\% CIs. The metaanalysis used the random effects (DerSimonian and Laird) model because of the anticipated heterogeneity across studies. Cochran's Q test and the $I^{2}$ statistic were used to quantify the presence of statistical heterogeneity (Higgins, Thompson, Deeks, \& Altman, 2003). $I^{2}$ values of $25 \%, 50 \%$, and $75 \%$ were considered to be low, moderate, and high degrees of heterogeneity, respectively (Higgins et al., 2003).

To explore potential sources of heterogeneity, subgroup analyses were undertaken based on the following characteristics: sex, study region (North America vs other), frailty measurement approach (physical phenotype vs other), sample size $(\leq 1,000$ vs $>1,000)$, duration of follow-up ( $\leq 2$ years vs $>2$ years), and country income level (highincome vs other). A high-income country (HIC) was defined using the World Bank criteria as any country with a gross national income per capita of USD 12,056 or more in 2017 (The World Bank, 2019). Differences between subgroups were assessed via a chi-square test (Sedgwick, 2015). We also pooled results specifically for studies that accounted for progression to death to examine its impact on the frailty and pre-frailty remission rates.

Publication bias was assessed using visual inspection of funnel plots and quantified via Egger's test (Egger, Davey Smith, Schneider, \& Minder, 1997).

For the baseline prevalence of frailty and pre-frailty, the meta-analysis was performed using the Freeman-Tukey double arcsine transformed proportions to stabilize the variance (Barendregt, Doi, Lee, Norman, \& Vos, 2013). If studies did not report baseline prevalence or lacked data to allow for calculations, they were excluded from this analysis.

All analyses were conducted using Stata 15/IC (StataCorp LP, College Station, Texas, USA). $p<.05$ was considered statistically significant.

\section{Results}

\section{Selection Processes}

A PRISMA flowchart illustrating the study selection process is presented in Figure 1. The electronic database search 
yielded 16,945 citations, of which 4,782 duplicates were excluded. A total of 12,048 articles were also excluded after title and abstract review, leaving 115 studies for potential inclusion. Following full-text assessment, 22 studies were found to meet the eligibility criteria. Three additional studies were retrieved from reference screening, resulting in a total of 25 studies included for the final review. No study was excluded on the basis of methodological (quality) evaluation using the JBI critical assessment tool for prevalence and incidence studies (Munn et al., 2015).

\section{Study Characteristics}

The characteristics of the 25 studies included in the review are summarized in Table 1 . The studies were conducted among a total of 31,336 older adults with frailty or prefrailty from 26 countries. In terms of the regional distribution of studies; six were from Asia, six from North America, three from South America, seven from Europe, two from Australia, and one cross-regional study. The study sample size varied from 50 to 8,913 (median $=463)$. There were considerable differences in the duration of follow-up, with the median being 3.0 (range 1-10.0) years.

Twenty studies measured frailty according to physical phenotype (using the Fried criteria or a modified version), three studies used the frailty index, one study used the Vulnerable Elders Survey-13 screening tool, and one study used both the Fried's criteria and frailty index. Among the included studies that used the deficit accumulation approach, the number of deficits used ranged from 20 to 44 .

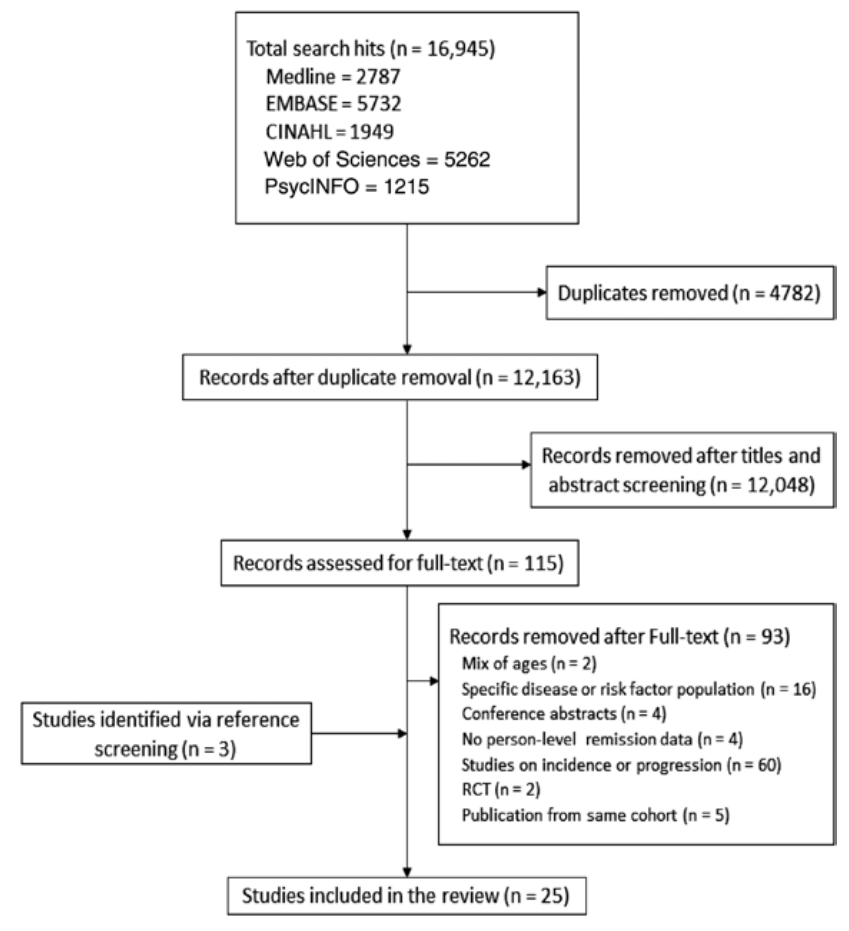

Figure 1. Flow chart of studies selection process.

\section{Prevalence of Frailty and Pre-frailty Among Baseline Source Population}

From a baseline source population of 52,312 older adults across 24 included studies, the pooled prevalence of frailty was $12.8 \%\left(95 \%\right.$ CI $\left.9.1-17.0 ; I^{2}=99.4 \%, p<.001\right)$ (Supplementary Figure 1). The pooled prevalence of frailty was lower among studies that measured frailty according to physical phenotype $\left(9.2 \%, 95 \%\right.$ CI $7.8-10.6 ; I^{2}=95.1, p$ $<.001)$ than studies using other methods $(37.0 \%, 95 \% \mathrm{CI}$ $\left.27.3-47.3 ; I^{2}=98.4, p<.001\right)$ ( $p$-value for difference <.001). The prevalence of frailty was also significantly lower among studies from HICs $\left(12.1 \%, 95 \%\right.$ CI 9.6-14.8; $I^{2}=98.0 \%, p$ $<.001)$ compared to those from non-HICs $(16.5 \%, 95 \%$ CI $\left.6.1-30.7 ; I^{2}=99.6, p<.001\right)$ ( $p$-value for difference $<.001$ ).

Data on baseline prevalence of pre-frailty were obtained from 21 studies. In these studies, involving 50,633 older adults at baseline, the pooled prevalence of pre-frailty was $50.5 \%$ (95\% CI 47.8-53.3; $I^{2}=97.1 \%, p<.001$ ) (Supplementary Figure 2). The pooled prevalence of pre-frailty was higher among studies that measured frailty according to physical phenotype $\left(51.3 \%, 95 \%\right.$ CI $\left.47.1-53.5 ; I^{2}=97.1 \%, p<.001\right)$ than studies using other criteria $(46.0 \%, 95 \%$ CI 34.7-57.5, $\left.I^{2}=98.5 \%, p<.001\right)$ ( $p$-value for difference <.001). There was no significant difference in the pooled prevalence of prefrailty between HICs $\left(50.2 \%, 95 \%\right.$ CI 47.1-54.3; $I^{2}=95.3$, $p<.001)$ and other countries $(51.1 \%, 95 \%$ CI $45.1-57.1$, $\left.I^{2}=97.4 \%, p<.001\right)(p$-value for difference $=.4120)$.

\section{Transition From Pre-frailty to Robust}

A total of 22 studies reported data on transitions among 23,869 people with pre-frailty at baseline. Of the 20,281 people with pre-frailty at baseline who were alive at the end of a median follow-up duration of 3.0 years, the proportion that regressed from pre-frailty to a robust state was $23.3 \%$. The pooled transition rate from pre-frailty to robust was 80.4 (95\% CI 61.7-104.6; $\left.I^{2}=98.6, p<.001\right)$ per 1,000 person-years (Figure 2). Visual inspection of funnel plots did not reveal any patterns of publication bias (Supplementary Figure 3), and this was confirmed via Egger's regression test $(p=.873)$.

Fourteen studies reported transitions to death among 19,749 pre-frail people. The proportion of participants who progressed from pre-frailty to death was $15.6 \%$ over a median follow-up of 3.5 years. When factoring in death, the pooled rate of regression from pre-frailty to robust was 51.9 (95\% CI 37.3-72.1; $\left.I^{2}=98.9, p<.001\right)$ per 1,000 person-years (Supplementary Figure 4). Restricting the analysis to survivors in these 14 studies resulted in a pooled transition rate from pre-frailty to robust of $61.8\left(95 \%\right.$ CI $47.5-80.3 ; I^{2}=98.2, p<$ .001 ) per 1,000 person-years (Supplementary Figure 5).

\section{Transition From Frailty to Pre-frailty or Robust}

A total of 24 studies reported data on transitions among 7,467 people with frailty at baseline. Of the 4,180 people with frailty who were alive at the end of a median follow-up 


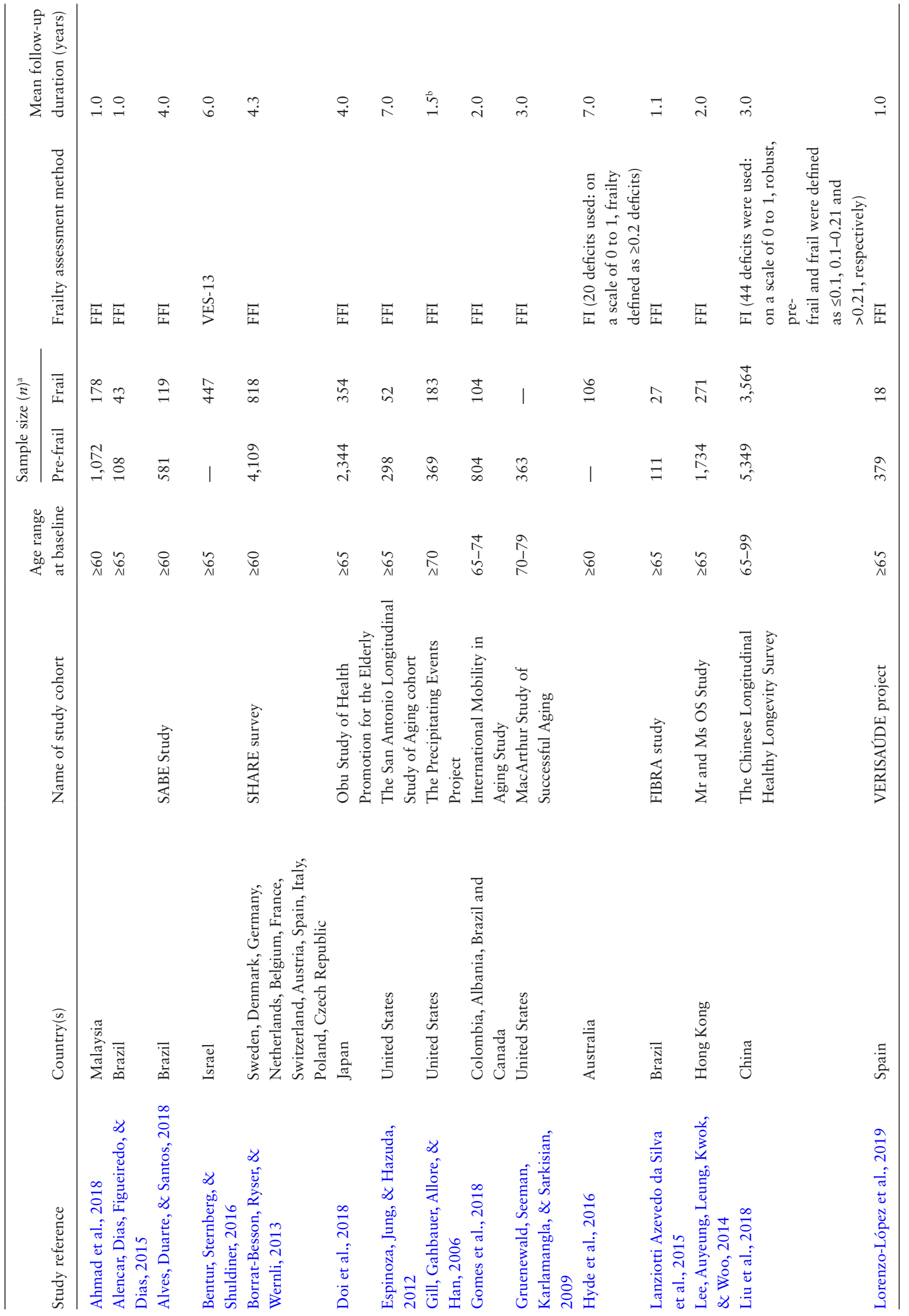




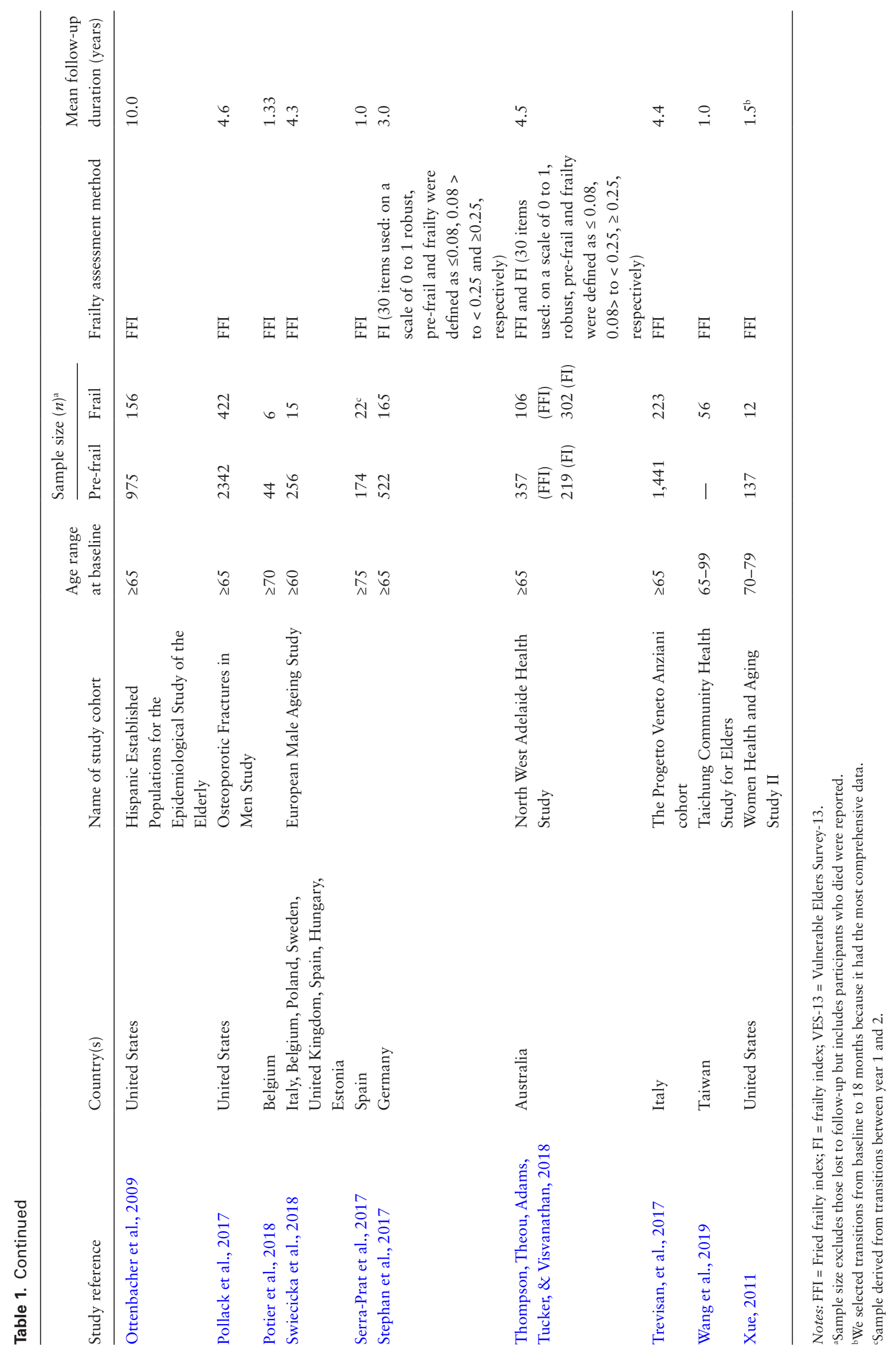




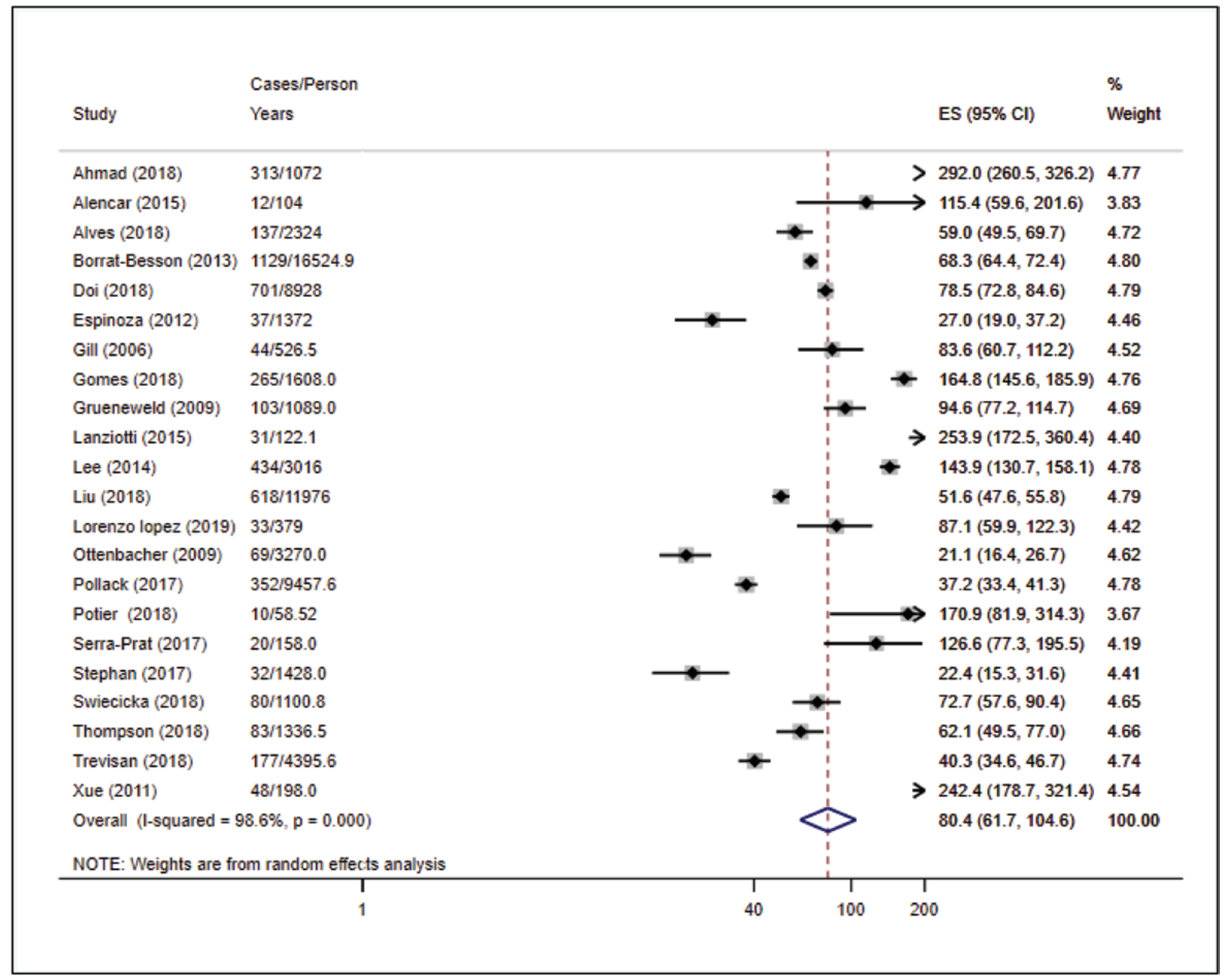

Figure 2. Forest plot of the rate of regression (per 1,000 person-years) from pre-frailty to robust state.

of 3.0 years, the proportion of people that transitioned from frailty to pre-frailty or a robust state was $35.2 \%$. Among the frail population, the pooled rate of remission was $135.3(95 \%$ CI 98.1-186.5; $\left.I^{2}=96.7, p<.001\right)$ per 1,000 person-years (Figure 3). Visual inspection of funnel plots did not reveal any patterns of publication bias (Supplementary Figure 6) and this was confirmed via Egger's regression test $(p=.686)$.

Sixteen studies reported transitions to death among 6,744 frail people. The proportion of participants who progressed from frailty to death was $45.8 \%$ over a median follow-up of 4.2 years. When factoring in death, the pooled rate of regression from frailty to pre-frailty or robust was $57.2\left(95 \%\right.$ CI 37.0-88.4; $\left.I^{2}=97.8, p<.001\right)$ per 1,000 person-years (Supplementary Figure 7). Restricting the analyses to those who survived in these 15 studies resulted in a pooled transition rate from frailty to prefrailty or robust of 98.5 (95\% CI 72.4-133.9; $I^{2}=95.2, p$ $<.001)$ per 1,000 person-years (Supplementary Figure 8).

\section{Subgroup and Sensitivity Analyses}

The pooled remission rates of frailty and pre-frailty were significantly higher across studies that measured frailty according to physical phenotype compared to those using other criteria (Table 2 ). Frailty and pre-frailty remission rates were significantly higher in women than men. The pooled regression rates of frailty and pre-frailty were also significantly higher in studies with shorter follow-up duration ( $\leq 2$ years). Pooled rates of frailty and pre-frailty remission were lower among studies involving participants from North America compared to studies involving participants from rest of the world. There was also variability in the pooled rates of frailty remission as per country income levels and study sample size. Nonetheless, the subgroup analyses did not reveal any consistent patterns in the source of heterogeneity as evidenced by the change in the $I^{2}$ statistic.

\section{Discussion}

In this systematic review and meta-analysis, we found that nearly one in four people who were pre-frail and more than one in three people who were frail and who survived over a median follow-up of 3.0 years regressed to a less severe frailty state. The remission rates among pre-frail and frail individuals were estimated as 80 and 135 cases per 1,000 


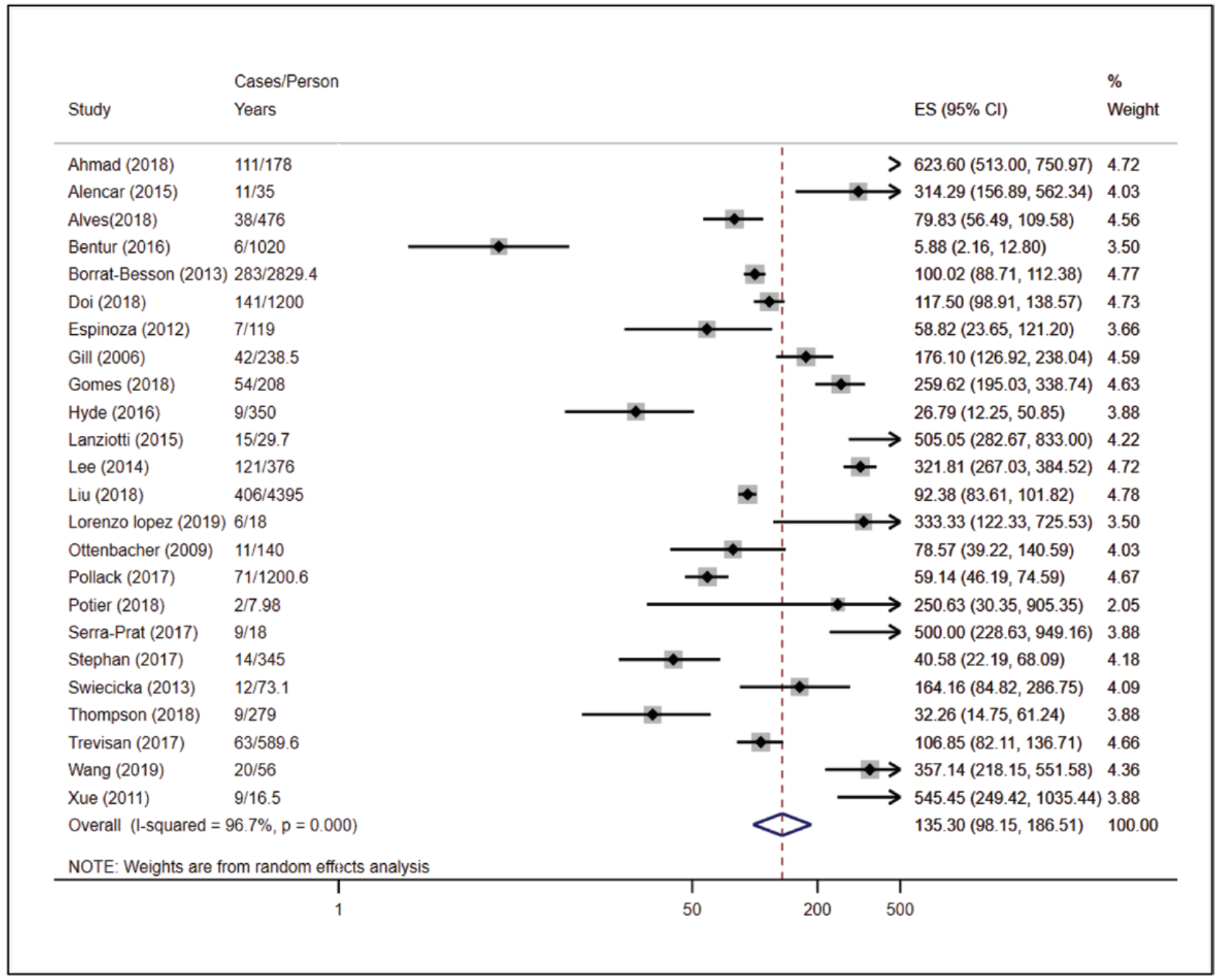

Figure 3. Forest plot of the rate of regression (per 1,000 person-years) from frailty to robust or pre-frail state.

person-years, respectively. These rates varied by gender, frailty assessment method, as well as by study characteristics such as the duration of follow-up.

Although not necessarily an inevitable consequence of aging, frailty is a problematic issue which is highly prevalent among older adults (Clegg et al., 2013; Xue, 2011). Across the studies included in this review, we found the prevalence of frailty and pre-frailty among the baseline source population to be $12.8 \%$ and $50.5 \%$, respectively. These estimates are higher than that reported from a previous systematic review by Collard and colleagues (2012). Nonetheless, the review by Collard and colleagues pooled data mainly from HICs, whereas our analyses combined data from countries with different income levels. A recent meta-analysis observed a much higher prevalence of pre-frailty $(49.3 \%)$ and frailty $(17.5 \%)$ in low and middle income countries (Siriwardhana, Hardoon, Rait, Weerasinghe, \& Walters, 2018). Indeed, among the studies included in this review, the pooled prevalence of frailty was significantly lower among studies from HICs than across studies from non-HICs.
The dynamic nature of frailty and the corresponding opportunity to transition between frailty states has been recognized (Lang et al., 2009; Michel et al., 2015; Xue, 2011). In particular, Kojima and colleagues, recently characterized various transition patterns among people aged at least 50 years with frailty based on the frailty phenotype (Kojima et al., 2019). They found that about $46 \%$ of frail individuals regressed to robust or pre-frail state over a median follow-up of 3.9 years. Our analysis showed that around $35.2 \%$ of frail older adults aged 60 years and older regressed if they survived a period of 3.0 years. The higher proportion of frail people experiencing remission in the analysis by Kojima and colleagues, may be partly due to their inclusion of younger people, because frailty remission has been found to decrease with increasing age (Trevisan et al., 2017). The current review has also estimated the rate of remission (per 1,000 person-years) that allows for better understanding of how quickly remission occurs (Jager et al., 2007). Furthermore, our study has characterized the frailty and pre-frailty remission rates 
Table 2. Subgroup Analyses of the Rate of Frailty and Pre-frailty Regression

\begin{tabular}{|c|c|c|c|c|c|}
\hline & $\begin{array}{l}\text { No. of } \\
\text { studies }^{\mathrm{a}}\end{array}$ & Sample size $\mathrm{e}^{\mathrm{b}}$ & $\begin{array}{l}\text { Pooled rate }(95 \% \mathrm{CI}) \\
\text { per } 1,000 \text { person-years }\end{array}$ & $I^{2}(p$-value $)$ & $\begin{array}{l}\chi^{2}, p \text {-value for } \\
\text { difference }^{c}\end{array}$ \\
\hline \multicolumn{6}{|l|}{ Pre-frailty to robust } \\
\hline \multicolumn{6}{|l|}{ Method of assessment } \\
\hline Physical phenotype & 20 & 15,813 & $87.6(65.9-116.4)$ & $98.6(<.001)$ & \multirow[t]{2}{*}{$16.2(<.001)$} \\
\hline Other & 3 & 4,662 & $41.8(27.0-64.8)$ & $90.3(<.001)$ & \\
\hline \multicolumn{6}{|l|}{ Sex } \\
\hline Male & 6 & 5,136 & $68.0(43.5-106.3)$ & $97.7(<.001)$ & \multirow[t]{2}{*}{$4.2(.040)$} \\
\hline Female & 5 & 4,037 & $94.1(53.5-165.7)$ & $98.3(<.001)$ & \\
\hline \multicolumn{6}{|l|}{ Study region } \\
\hline North America & 6 & 3,425 & $58.5(31.1-110.1)$ & $98.0(<.001)$ & \multirow[t]{2}{*}{$5.5(.019)$} \\
\hline Other & 15 & 1,6052 & $86.8(65.5-116.9)$ & $98.6(<.001)$ & \\
\hline \multicolumn{6}{|l|}{ Mean follow-up period } \\
\hline$\leq 2$ years & 10 & 4663 & $157.2(119.9-206.0)$ & $93.8(<.001)$ & \multirow[t]{2}{*}{$57.3(<.001)$} \\
\hline$>2$ years & 12 & 15,618 & $48.6(39.8-59.4)$ & $96.7(<.001)$ & \\
\hline \multicolumn{6}{|l|}{ Sample size } \\
\hline$\leq 1,000$ & 16 & 5,578 & $78.2(54.2-112.9)$ & $97.2(<.001)$ & \multirow[t]{2}{*}{$0.45(.4983)$} \\
\hline$>1,000$ & 6 & 14,703 & $86.9(53.7-140.6)$ & $99.5(<.001)$ & \\
\hline \multicolumn{6}{|l|}{ Country income level } \\
\hline $\mathrm{HIC}$ & 16 & 13,617 & $67.4(52.2-87.1)$ & $97.8(<.001)$ & \multirow[t]{2}{*}{$15.1(<.001)$} \\
\hline Other & 5 & 5,860 & 120.7 (48.9-297.8) & $99.4(<.001)$ & \\
\hline \multicolumn{6}{|c|}{ Frailty to pre-frailty or robust } \\
\hline \multicolumn{6}{|c|}{ Method of assessment } \\
\hline Physical phenotype & 20 & 2,382 & $160.2(150.4-170.5)$ & $96.3(<.001)$ & \multirow[t]{2}{*}{$26.4(<.001)$} \\
\hline Other & 5 & 2,009 & $80.5(73.3-88.3)$ & $95.2(<.001)$ & \\
\hline \multicolumn{6}{|l|}{ Sex } \\
\hline Male & 6 & 591 & $134.9(79.8-231.7)$ & $98.1(<.001)$ & \multirow[t]{2}{*}{$6.7(.01)$} \\
\hline Female & 5 & 686 & $181.1(82.3-398.0)$ & $96.9(<.001)$ & \\
\hline \multicolumn{6}{|l|}{ Study region } \\
\hline North America & 5 & 462 & $120.2(57.3-252.1)$ & $92.7(<.001)$ & \multirow[t]{2}{*}{$2.2(.1399)$} \\
\hline Other & 18 & 3,614 & $144.2(91.8-196.2)$ & $97.2(<.001)$ & \\
\hline \multicolumn{6}{|l|}{ Mean follow-up period } \\
\hline$\leq 2$ years & 11 & 800 & $358.3(265.2-484.1)$ & $84.0(<.001)$ & \multirow[t]{2}{*}{$195.1(<.001)$} \\
\hline$>2$ years & 13 & 3,380 & $69.4(55.8-86.4)$ & $87.3(<.001)$ & \\
\hline \multicolumn{6}{|l|}{ Sample size } \\
\hline$\leq 1,000$ & 23 & 2,715 & $137.4(95.9-196.9)$ & $96.4(<.001)$ & \multirow[t]{2}{*}{$-^{\mathrm{d}}$} \\
\hline$>1,000$ & 1 & 1,465 & $92.4(83.6-101.8)$ & - & \\
\hline \multicolumn{6}{|l|}{ Country income level } \\
\hline $\mathrm{HIC}$ & 18 & 2,252 & $110.5(78.1-156.5)$ & $94.6(<.001)$ & \multirow[t]{2}{*}{$44.1(<.001)$} \\
\hline Other & 5 & 1,824 & $233.7(83.9-651.2)$ & $98.8(<.001)$ & \\
\hline
\end{tabular}

Notes: $\mathrm{CI}=$ confidence interval; HIC = high-income economy.

a Some studies were excluded from subgroup or may fit under more than one subgroup and thus the total number of studies in a subgroup analysis may exceed the total number of included studies.

bThe results presented in this table are per the fully surviving cohort in each study.

c $p$-values derived via chi-square test.

${ }^{\mathrm{d}}$ Statistical significance not assessed.

across different diagnostic criteria and shown that the rates of remission are significantly higher when using the frailty phenotype than other methods. This observation further supports the growing calls for a harmonized approach to frailty assessment to improve the precision in frailty data. However, overall, the study results are in accord with those of prior studies (Kojima et al., 2019; O'Caoimh et al., 2018), demonstrating that natural remission of frailty is possible and that individuals classified as frail are not necessarily in an irreversible health condition.

Several factors such as low physical activity (Ahmad et al., 2018; Trevisan et al., 2017), polypharmacy (including the use of medications such as sedatives and anticholinergic agents) (Lorenzo-López et al., 2019; Thompson et al., 2018), obesity (Thompson et al., 2018), underweight (Trevisan et al., 2017), presence of comorbidities such as stroke, diabetes (Espinoza et al., 2012; Lee et al., 2014; 
Trevisan et al., 2017), smoking, vision or hearing loss (Trevisan et al., 2017), and cognitive impairment (Trevisan et al., 2017), have been identified to reduce the likelihood of frailty regression. This highlights that efforts to address these comorbidities may help to reduce the burden of frailty.

Previous studies have shown higher burden of frailty among females than males across all age groups (Collard et al., 2012; Gordon et al., 2017). Nonetheless, our pooled data suggested that frailty and pre-frailty remission is more common in females than in males. The contributory factors to the higher rate of frailty and pre-frailty remission in females deserves further exploration.

Owing to the adverse health outcomes and costs associated with frailty (Bock et al., 2016; Cheng \& Chang, 2017; Kojima, 2015), better understanding of the patterns and determinants of frailty is important. By characterizing the rate of frailty remission, our study contributes to improving the understanding of the natural trajectory of frailty among community dwelling older adults. Such information is likely to be useful to professionals involved in the delivery of health care to the geriatric population who may want to know about possible outcomes of frailty in the absence of interventions.

As frailty improvement has been shown to be possible, the timely implementation of appropriate interventions could facilitate remission among pre-frail and frail individuals and potentially avert adverse-related consequences. For example, several interventions incorporating physical exercise, health education, nutritional supplements, home visits, hormone supplements, and counselling have been evaluated for their potential to delay or reverse frailty (Apostolo et al., 2018; Chin, van Uffelen, Riphagen, \& van Mechelen, 2008; de Labra, Guimaraes-Pinheiro, Maseda, Lorenzo, \& Millan-Calenti, 2015; Giné-Garriga, Roque-Figuls, CollPlanas, Sitja-Rabert, \& Salva, 2014; Puts et al., 2017), and highlighted in contemporary guidelines (Dent et al., 2017).

Recently, Travers, Romero-Ortuno, Bailey, and Cooney (2019) also compared the relative effectiveness and ease of implementation of the various interventions to delay or reverse frailty specifically within primary care settings. They found that interventions with both strength training and protein supplementation ranked highest in terms of relative effectiveness and ease of implementation. Although relatively easy to implement, interventions with mild-intensity mixed exercises or singular exercises such as walking ranked in the mid-zone for relative effectiveness (Travers et al., 2019). Similarly, educational or health promotion activities typically placed in the mid-zone for both relative effectiveness as well as ease of implementation. On the other hand, interventions targeting behavioral change ranked low in relative effectiveness and the mid-zone for ease of implementation. Whereas comprehensive geriatric assessments and home visits ranked mid-low for both relative effectiveness as well as ease of implementation (Travers et al., 2019).

Our review has some strengths. We included a large number of studies from multiple countries, with no evidence of publication bias. By not restricting our analyses to any specific frailty assessment method, the results provide a broader context for understanding the patterns of remission among people with frailty. In addition, for the main analysis, we recalculated the remission rate in studies that incorporated deaths to allow for comparison to studies that used fully surviving cohorts. This approach was important to reduce the impact of survivorship bias (DelgadoRodriguez \& Llorca, 2004). Indeed, a subsequent subgroup analyses showed that incorporation of transition to deaths had significant impact on the remission rates. This was particularly pronounced for frailty than pre-frailty given that transition to deaths are more frequent in frail than prefrail individuals (Chang \& Lin, 2015; Kojima et al., 2018; Vermeiren et al., 2016).

In terms of the limitations of our study, there was substantial heterogeneity unexplained by subgroup analyses and largely reflecting methodological differences. In addition, we could not stratify results according to age groups because of limited number of studies reporting age-stratified data. Moreover, this review was focused on the proportion of people who experienced remission rather than the number of transitions. Because an individual may undergo multiple intermediary transitions (Jamsen et al., 2016; Kojima, Taniguchi, Iliffe, Jivraj, \& Walters, 2019; O'Caoimh et al., 2018), our analytic framework did not fully capture the full extent of frailty transitions.

\section{Conclusions}

Although frailty is highly prevalent among older adults, it is a dynamic syndrome, and natural remission is not only possible, but common. Improved understanding of the factors that confer increased likelihood of remission may support the design of interventions to reduce the burden of frailty in an era of population aging.

\section{Supplementary Material}

Supplementary data are available at The Gerontologist online.

\section{Funding}

None reported.

\section{Acknowledgement}

We are grateful to study authors who provided us with additional data.

\section{Conflict of Interest}

The authors declare no potential conflicts of interest. 


\section{References}

Aguayo, G. A., Donneau, A. F., Vaillant, M. T., Schritz, A., Franco, O. H., Stranges, S., . . Witte, D. R. (2017). Agreement between 35 published frailty scores in the general population. American Journal of Epidemiology, 186, 420-434. doi:10.1093/ aje/kwx061

Ahmad, N. S., Hairi, N. N., Said, M. A., Kamaruzzaman, S. B., Choo, W. Y., Hairi, F., . . . Bulgiba, A. (2018). Prevalence, transitions and factors predicting transition between frailty states among rural community-dwelling older adults in Malaysia. PLoS One, 13, e0206445. doi:10.1371/journal. pone. 0206445

Alencar, M. A., Dias, J. M. D., Figueiredo, L. C., \& Dias, R. C. (2015). Transitions in frailty status in community-dwelling older adults. Topics in Geriatric Rehabilitation, 31, 105-112. doi:10.1097/TGR.0000000000000055

Alves, L., Duarte, Y., \& Santos, J. (2018). Factors Associated the Transitions in the Frailty States Among Elderly in Brazil, 2006-2010. Paper presented at: the Population Association of America, 2018 Annual Meeting. Retrieved from https://paa. confex.com/paa/2018/meetingapp.cgi/Paper/20340

Ambagtsheer, R. C., Beilby, J. J., Visvanathan, R., Dent, E., Yu, S., \& Braunack-Mayer, A. J. (2019). Should we screen for frailty in primary care settings? A fresh perspective on the frailty evidence base: A narrative review. Preventive Medicine, 119, 63-69. doi:10.1016/j.ypmed.2018.12.020

Apostolo, J., Cooke, R., Bobrowicz-Campos, E., Santana, S., Marcucci, M., Cano, A., . . . Holland, C. (2018). Effectiveness of interventions to prevent pre-frailty and frailty progression in older adults: A systematic review. JBI Database of Systematic Reviews Implementation Reports, 16, 140-232. doi:10.11124/ JBISRIR-2017-003382.m

Bandeen-Roche, K. \& Espinoza, S. E. (2017). Getting underneath observed frailty transitions: Meaningful change, prevention, and intervention. Journal of the American Geriatrics Society, 65, 2455-2456. doi:10.1111/jgs.15034

Barendregt, J. J., Doi, S. A., Lee, Y. Y., Norman, R. E., \& Vos, T. (2013). Meta-analysis of prevalence. Journal of Epidemiology and Community Health, 67, 974-978. doi:10.1136/ jech-2013-203104

Bentur, N., Sternberg, S. A., \& Shuldiner, J. (2016). Frailty transitions in community dwelling older people. The Israel Medical Association Journal, 18, 449-453.

Bock, J. O., König, H. H., Brenner, H., Haefeli, W. E., Quinzler, R., Matschinger, H., \& Heider, D (2016). Associations of frailty with health care costs-results of the ESTHER cohort study. BMC Health Services Research, 16, 128. doi:10.1186/ s12913-016-1360-3

Borrat-Besson, C., Ryser, V., \& Wernli, B. (2013). Transitions between frailty states-a European comparison. In A. BörschSupan, M. Brandt, H. Litwin \& G. Weber (Eds.), Active ageing and solidarity between generations in Europe: First results from SHARE after the economic crisis. (pp. 175-185). Germany: Hubert \& Co. GmBH \& Co. KG.

Cesari, M., Gambassi, G., van Kan, G. A., \& Vellas, B. (2014). The frailty phenotype and the frailty index: Different instruments for different purposes. Age and Ageing, 43, 10-12. doi:10.1093/ ageing/aft160
Chang, S. F., \& Lin, P. L. (2015). Frail phenotype and mortality prediction: A systematic review and meta-analysis of prospective cohort studies. International journal of Nursing Studies, 52, 1362-1374. doi:10.1016/j.ijnurstu.2015.04.005

Chang, S. F., Lin, H. C., \& Cheng, C. L. (2018). The relationship of frailty and hospitalization among older people: Evidence from a meta-analysis. Journal of Nursing Scholarship, 50, 383-391. doi:10.1111/jnu.12397

Cheng, M. H., \& Chang, S. F. (2017). Frailty as a risk factor for falls among community dwelling people: Evidence from a meta-analysis. Journal of Nursing Scholarship, 49, 529-536. doi:10.1111/jnu.12322

Chin, A. P. M. J., van Uffelen, J. G., Riphagen, I., \& van Mechelen, W. (2008). The functional effects of physical exercise training in frail older people: A systematic review. Sports Medicine, 38, 781-793. doi: 10.2165/00007256-200838090-00006

Clegg, A., Young, J., Iliffe, S., Rikkert, M. O., \& Rockwood, K. (2013). Frailty in elderly people. Lancet (London, England), 381, 752-762. doi:10.1016/S0140-6736(12)62167-9

Collard, R. M., Boter, H., Schoevers, R. A., \& Oude Voshaar, R. C. (2012). Prevalence of frailty in community-dwelling older persons: A systematic review. Journal of the American Geriatrics Society, 60, 1487-1492. doi:10.1111/j.1532-5415.2012.04054.x

Delgado-Rodriguez, M., \& Llorca, J. (2004). Bias. Journal of Epidemiology and Community Health, 58, 635-641. doi:10.1136/jech.2003.008466

Dent, E., Kowal, P., \& Hoogendijk, E. O. (2016). Frailty measurement in research and clinical practice: A review. European Journal of Internal Medicine, 31, 3-10. doi:10.1016/j.ejim.2016.03.007

Dent, E., Lien, C., Lim, W. S., Wong, W. C., Wong, C. H., Ng, T. P., ... Flicker, L (2017). The Asia-Pacific clinical practice guidelines for the management of frailty. Journal of the American Medical Directors Association, 18, 564-575. doi:10.1016/j. jamda.2017.04.018

Department of Economic and Social Affairs Population Division. (2013). World Population Ageing 2013. ST/ESA/SER.A/348. New York: UN.

Doi, T., Makizako, H., Tsutsumimoto, K., Nakakubo, S., Kim, M. J., Kurita, S., ... Shimada, H. (2018). Transitional status and modifiable risk of frailty in Japanese older adults: A prospective cohort study. Geriatrics and Gerontology International, 18, 1562-1566. doi:10.1111/ggi.13525

Egger, M., Davey Smith, G., Schneider, M., \& Minder, C. (1997). Bias in meta-analysis detected by a simple, graphical test. BMJ, 315, 629-634. doi:doi.org/10.1136/bmj.315.7109.629

Espinoza, S. E., Jung, I., \& Hazuda, H. (2012). Frailty transitions in the San Antonio longitudinal study of aging. Journal of the American Geriatrics Society, 60, 652-660. doi:10.1111/j.1532-5415.2011.03882.x

Fried, L. P., Tangen, C. M., Walston, J., Newman, A. B., Hirsch, C., Gottdiener, J., . . . McBurnie, M. A.; Cardiovascular Health Study Collaborative Research Group. (2001). Frailty in older adults: Evidence for a phenotype. The Journal of Gerontology Series A: Biological Sciences and Medical Sciences, 56, M146156. doi:10.1093/gerona/56.3.M146

Gill, T. M., Gahbauer, E. A., Allore, H. G., \& Han, L. (2006). Transitions between frailty states among community-living 
older persons. Archives of Internal Medicine, 166, 418-423. doi:10.1001/archinte.166.4.418

Giné-Garriga, M., Roqué-Fíguls, M., Coll-Planas, L., SitjàRabert, M., \& Salvà, A. (2014). Physical exercise interventions for improving performance-based measures of physical function in community-dwelling, frail older adults: A systematic review and meta-analysis. Archives of Physical Medicine and Rehabilitation, 95, 753-769.e3. doi:10.1016/j.apmr.2013.11.007

Gomes, C. D. S., Guerra, R. O., Wu, Y. Y., Barbosa, J. F. S., Gomez, F., Sousa, A., \& Pirkle, C. M. (2018). Social and economic predictors of worse frailty status occurrence across selected countries in North and South America and Europe. Innovation in Aging, 2, 1-8. doi:10.1093/geroni/igy037

Gordon, E. H., Peel, N. M., Samanta, M., Theou, O., Howlett, S. E., \& Hubbard, R. E. (2017). Sex differences in frailty: A systematic review and meta-analysis. Experimental Gerontology, 89, 30-40. doi:10.1016/j.exger.2016.12.021

Gruenewald, T. L., Seeman, T. E., Karlamangla, A. S., \& Sarkisian, C. A. (2009). Allostatic load and frailty in older adults. Journal of the American Geriatrics Society, 57, 15251531. doi:10.1111/j.1532-5415.2009.02389.x

Higgins, J. P., Thompson, S. G., Deeks, J. J., \& Altman, D. G. (2003). Measuring inconsistency in meta-analyses. BMJ (Clinical Research ed.), 327, 557-560. doi:10.1136/bmj.327.7414.557

Hubbard, R. E., O’Mahony, M. S., \& Woodhouse, K. W. (2009). Characterising frailty in the clinical setting-a comparison of different approaches. Age and Ageing, 38, 115-119. doi:10.1093/ ageing/afn252

Hyde, Z., Flicker, L., Smith, K., Atkinson, D., Fenner, S., Skeaf, L., . . Lo Giudice D (2016). Prevalence and incidence of frailty in Aboriginal Australians, and associations with mortality and disability. Maturitas, 87, 89-94. doi:10.1016/j. maturitas.2016.02.013

Jager, K. J., Zoccali, C., Kramar, R., \& Dekker, F. W. (2007). Measuring disease occurrence. Kidney Int, 72, 412-415. doi: 10.1038/sj.ki.5002341

Jamsen, K. M., Bell, J. S., Hilmer, S. N., Kirkpatrick, C. M., Ilomäki, J., Le Couteur, D., . . G Gnjidic, D. (2016). Effects of changes in number of medications and drug burden index exposure on transitions between frailty states and death: The concord health and ageing in men project cohort study. Journal of the American Geriatrics Society, 64, 89-95. doi:10.1111/ jgs. 13877

Kojima, G. (2015). Frailty as a predictor of future falls among community-dwelling older people: A systematic review and meta-analysis. Journal of the American Medical Directors Association, 16, 1027-1033. doi:10.1016/j.jamda.2015.06.018

Kojima, G. (2016). Frailty as a predictor of hospitalisation among community-dwelling older people: A systematic review and meta-analysis. Journal of Epidemiology and Community Health, 70, 722-729. doi:10.1136/jech-2015-206978

Kojima, G. (2017). Frailty as a predictor of disabilities among community-dwelling older people: A systematic review and meta-analysis. Disability and Rehabilitation, 39, 1897-1908. doi:10.1080/09638288.2016.1212282

Kojima, G. (2018). Frailty as a predictor of nursing home placement among community-dwelling older adults: A systematic review and meta-analysis. Journal of Geriatric Physical Therapy, 41, 42-48. doi:10.1519/JPT.0000000000000097
Kojima, G., Iliffe, S., \& Walters, K. (2018). Frailty index as a predictor of mortality: A systematic review and meta-analysis. Age and Ageing, 47, 193-200. doi:10.1093/ageing/afx162

Kojima, G., Taniguchi, Y., Iliffe, S., Jivraj, S., \& Walters, K. (2019). Transitions between frailty states among community-dwelling older people: A systematic review and meta-analysis. Ageing Research Reviews, 50, 81-88. doi:10.1016/j.arr.2019.01.010

de Labra, C., Guimaraes-Pinheiro, C., Maseda, A., Lorenzo, T., \& Millán-Calenti, J. C. (2015). Effects of physical exercise interventions in frail older adults: A systematic review of randomized controlled trials. BMC Geriatrics, 15, 154. doi:10.1186/s12877-015-0155-4

Lang, P. O., Michel, J. P., \& Zekry, D. (2009). Frailty syndrome: A transitional state in a dynamic process. Gerontology, 55, 539549. doi:10.1159/000211949

Lanziotti Azevedo da Silva, S., Campos Cavalcanti Maciel, A., de Sousa Maximo Pereira, L., Domingues Dias, J. M., Guimaraes de Assis, M., \& Correa Dias, R. (2015). Transition patterns of frailty syndrome in comunity-dwelling elderly individuals: A longitudinal study. Journal of Frailty and Aging, 4, 50-55. doi:10.14283/jfa.2015.43

Lee, J. S. W., Auyeung, T.-W., Leung, J., Kwok, T., \& Woo, J. (2014). Transitions in frailty states among community-living older adults and their associated factors. Journal of the American Medical Directors Association, 15, 281-286. doi:10.1016/j. jamda.2013.12.002

Liu, Z. Y., Wei, Y. Z., Wei, L. Q., Jiang, X. Y., Wang, X. F., Shi, Y., \& Hai, H. (2018). Frailty transitions and types of death in Chinese older adults: A population-based cohort study. Clinical Interventions in Aging, 13, 947-956. doi:10.2147/CIA.S157089

Lorenzo-López, L., López-López, R., Maseda, A., Buján, A., Rodríguez-Villamil, J. L., \& Millán-Calenti, J. C. (2019). Changes in frailty status in a community-dwelling cohort of older adults: The VERISAÚDE study. Maturitas, 119, 54-60. doi:10.1016/j.maturitas.2018.11.006

Malmstrom, T. K., Miller, D. K., \& Morley, J. E. (2014). A comparison of four frailty models. Journal of the American Geriatrics Society, 62, 721-726. doi:10.1111/jgs.12735

Michel, J. P., Cruz-Jentoft, A. J., \& Cederholm, T. (2015). Frailty, exercise and nutrition. Clinics in Geriatric Medicine, 31, 375-387. doi:10.1016/j.cger.2015.04.006

Moher, D., Liberati, A., Tetzlaff, J., Altman, D. G., \& Group, P. (2009). Preferred reporting items for systematic reviews and meta-analyses: The PRISMA statement. BMJ, 339, b2535. doi:10.1371/journal.pmed.1000097.

Munn, Z., Moola, S., Lisy, K., Riitano, D., \& Tufanaru, C. (2015). Methodological guidance for systematic reviews of observational epidemiological studies reporting prevalence and cumulative incidence data. International Journal of Evidence-Based Healthcare, 13,147-153. doi:10.1097/XEB.0000000000000054

O’Caoimh, R., Galluzzo, L., Rodriguez-Laso, A., Van der Heyden, J., Ranhoff, A. H., Carcaillon-Bentata, L., \& Liew A.; Work Package 5 of the Joint Action ADVANTAGE (2018). Transitions and trajectories in frailty states over time: A systematic review of the European Joint Action ADVANTAGE. Annali dell'Istituto Superiore di Sanità, 54, 246-252. doi:10.4415/ANN_18_03_12 Ottenbacher, K. J., Graham, J. E., Al Snih, S., Raji, M., SamperTernent, R., Ostir, G. V., \& Markides, K. S. (2009). Mexican Americans and frailty: Findings from the Hispanic established 
populations epidemiologic studies of the elderly. American Journal of Public Health, 99, 673-679. doi:10.2105/ AJPH.2008.143958

Persico, I., Cesari, M., Morandi, A., Haas, J., Mazzola, P., Zambon, A., . . . Bellelli, G (2018). Frailty and delirium in older adults: A systematic review and meta-analysis of the literature. Journal of the American Geriatrics Society, 66, 2022-2030. doi:10.1111/jgs.15503

Pollack, L. R., Litwack-Harrison, S., Cawthon, P. M., Ensrud, K., Lane, N. E., Barrett-Connor, E., \& Dam, T. T. (2017). Patterns and predictors of frailty transitions in older men: The osteoporotic fractures in men study. Journal of the American Geriatrics Society, 65, 2473-2479. doi:10.1111/jgs.15003

Potier, F., Degryse, J. M., Bihin, B., Debacq-Chainiaux, F., CharletRenard, C., Martens, H., \& de Saint-Hubert, M. (2018). Health and frailty among older spousal caregivers: An observational cohort study in Belgium. BMC Geriatrics, 18, 291. doi:10.1186/ s12877-018-0980-3

Puts, M. T. E., Toubasi, S., Andrew, M. K., Ashe, M. C., Ploeg, J., Atkinson, E., . . . McGilton K.(2017). Interventions to prevent or reduce the level of frailty in community-dwelling older adults: A scoping review of the literature and international policies. Age and Ageing, 46, 383-392. doi:10.1093/ageing/afw247

Rockwood, K., Stadnyk, K., MacKnight, C., McDowell, I., Hébert, R., \& Hogan, D. B. (1999). A brief clinical instrument to classify frailty in elderly people. Lancet (London, England), 353, 205-206. doi:10.1016/S0140-6736(98)04402-X

Sedgwick, P. (2015). Meta-analyses: What is heterogeneity? BMJ (Clinical research ed.), 350, h1435. doi:10.1136/bmj.h1435

Serra-Prat, M., Papiol, M., Vico, J., Palomera, E., Arus, M., \& Cabre, M. (2017). Incidence and risk factors for frailty in the community-dwelling elderly population. A two-year follow-up cohort study. Journal of Gerontology and Geriatrics, 6, 1-8. doi:10.4172/2167-7182.1000452

Siriwardhana, D. D., Hardoon, S., Rait, G., Weerasinghe, M. C., \& Walters, K. R. (2018). Prevalence of frailty and prefrailty among community-dwelling older adults in low-income and middleincome countries: A systematic review and meta-analysis. BMJ Open, 8, e018195. doi:10.1136/bmjopen-2017-018195

Stephan, A. J., Strobl, R., Holle, R., Meisinger, C., Schulz, H., Ladwig, K. H., . . G Grill, E. (2017). Male sex and poverty predict abrupt health decline: Deficit accumulation patterns and trajectories in the KORA-Age cohort study. Preventive Medicine, 102, 31-38. doi:10.1016/j.ypmed.2017.06.032

Sutton, A., Abrams, K., Jones, D., Sheldon, T., \& Song, F. (2000). Methods for Meta-Analysis in Medical Research. London: Wiley.
Swiecicka, A., Eendebak, R., Lunt, M., O’Neill, T. W., Bartfai, G., Casanueva, F. F., ... Rutter, M. K; European Male Ageing Study Group. (2018). Reproductive hormone levels predict changes in frailty status in community-dwelling older men: European male ageing study prospective data. The Journal of Clinical Endocrinology and Metabolism, 103, 701-709. doi:10.1210/ jc.2017-01172

The World Bank. (2019). World Bank Country and Lending Groups. Retrieved January 29, 2019, from https://datahelpdesk.worldbank.org/knowledgebase/ articles/906519-world-bank-country-and-lending-groups

Thompson, M. Q., Theou, O., Adams, R. J., Tucker, G. R., \& Visvanathan, R. (2018). Frailty state transitions and associated factors in South Australian older adults. Geriatrics and Gerontology International, 18, 1549-1555. doi:10.1111/ggi.13522

Travers, J., Romero-Ortuno, R., Bailey, J., \& Cooney, M. T. (2019). Delaying and reversing frailty: A systematic review of primary care interventions. British Journal of General Practice, 69, e61e69. doi:10.3399/bjgp18X700241

Trevisan, C., Veronese, N., Maggi, S., Baggio, G., Toffanello, E. D., Zambon, S., .. . Sergi, G. (2017). Factors influencing transitions between frailty states in elderly adults: The Progetto Veneto Anziani Longitudinal Study. Journal of the American Geriatrics Society, 65, 179-184. doi:10.1111/jgs.14515

Turner, G., Clegg, A., British Geriatrics, S., Age, U. K., \& Royal College of General, P. (2014). Best practice guidelines for the management of frailty: A British Geriatrics Society, Age UK and Royal College of General Practitioners report. Age and Ageing, 43, 744-747. doi:10.1093/ageing/afu138

Vermeiren, S., Vella-Azzopardi, R., Beckwee, D., Habbig, A. K., Scafoglieri, A., Jansen, B., Bautmans I; Gerontopole Brussels Study group. (2016). Frailty and the prediction of negative health outcomes: A meta-analysis. Journal of the American Medical Directors Association, 17, 1163 e1161-1163 e1117. doi:10.1016/j.jamda.2016.09.010

Wang, M.C., Li, T. C., Li, C. I, Liu, C. S., Lin, W. Y., Lin, C., . . . Lin, C. C. (2019). Frailty, transition in frailty status and all-cause mortality in older adults of a Taichung community-based population. BMC Geriatrics, 19, 1-8. doi:10.1186/s12877-019-1039-9

Xue, Q. L. (2011). The frailty syndrome: Definition and natural history. Clinics in Geriatric Medicine, 27, 1-15. doi:10.1016/j. cger.2010.08.009

Yousef, F., Cardwell, C., Cantwell, M. M., Galway, K., Johnston, B. T., \& Murray, L. (2008). The incidence of esophageal cancer and high-grade dysplasia in Barrett's esophagus: A systematic review and meta-analysis. American Journal of Epidemiology, 168, 237-249. doi:10.1093/aje/kwn121 